\title{
Touchless Selection Schemes for Intelligent Automotive User Interfaces With Predictive Mid-Air Touch
}

\author{
Bashar I. Ahmad, University of Cambridge, Cambridge, UK \\ Chrisminder Hare, Jaguar Land Rover, Coventry, UK \\ Harpreet Singh, Jaguar Land Rover, Coventry, UK \\ Arber Shabani, Jaguar Land Rover, Coventry, UK \\ Briana Lindsay, Jaguar Land Rover, Coventry, UK \\ Lee Skrypchuk, Jaguar Land Rover, Coventry, UK \\ Patrick Langdon, University of Cambridge, Cambridge, UK \\ Simon Godsill, University of Cambridge, Cambridge, UK
}

\begin{abstract}
Predictive touch technology aims to improve the usability and performance of in-vehicle displays under the influence of perturbations due to the road and driving conditions. It fundamentally relies on predicting and early in the freehand pointing movement, the interface item the user intends to select, using a novel Bayesian inference framework. This article focusses on evaluating facilitation schemes for selecting the predicted interface component whilst driving, and without physically touching the display, thus touchless. Initially, several viable schemes were identified in a brainstorming session followed by an expert workshop with 12 participants. A simulator study with 24 participants using a prototype predictive touch system was then conducted. A number of collected quantitative and qualitative measures show that immediate mid-air selection, where the system autonomously autoselects the predicted interface component, may be the most promising strategy for predictive touch.
\end{abstract}

\section{KEYWORDS}

Automotive Interface, Bayesian Inference, Human Machine Interaction, Intelligent Interface, Pointing, Predictive Touch

\section{INTRODUCTION}

Predictive touch is an emerging HMI technology that employs a probabilistic Bayesian framework and novel algorithms to predict the interface component the user intends to select, notably early in the pointing-selection task (Ahmad, Murphy, Godsill, Langdon \& Hardy, 2017). It infers the user intent from the available freehand pointing movements in $3 \mathrm{D}$, for example from gesture trackers which are increasingly becoming commonplace in vehicles (Zhang \& Angell, 2014; 
Ohn-Bar \& Trivedi, 2014), and potentially other available sensory data such as eye-gaze. The pointing-selection task is simplified and expedited by the system via applying a suitable selection facilitation scheme. This can significantly reduce the effort and distractions associated with using in-vehicle displays whilst driving (Jæger, Skov \& Thomassen, 2008). Figure 1 depicts the system block diagram including the sensory data sources utilized by a Bayesian predictor to estimate, early in the pointing task, the probability of each of the selectable interface items being the intended on-screen destination. Predictive touch was originally developed to mitigate the effects of perturbations on the user input, for example vibrations and accelerations due to the road and driving conditions. They can have a detrimental impact on the performance of interactive displays, such as touch screens (Goode, Lenné \& Salmon, 2012; Ahmad et al., 2015), which often act as the gateway to control in-vehicle infotainment systems and are an integrated part of modern vehicles (Harvey \& Stanton, 2016).

With predictive touch, which is not a pointing/ray-casting or conventional "symbolic" gestures recognition solution; as will be discussed, the user does not need to physically touch a display to select an interface component. Therefore, this touchless technology can not only improve the usability and performance of in-vehicle interactive displays, but it also provides the means to interact via the intuitive free-hand pointing with new automotive display technologies that do not have a physical surface such as head-up displays and 3D projections (Bark et al., 2014; Broy et al., 2015). It also offers additional design flexibilities in terms of the display placement and size which is otherwise limited by the reach of the driver/ passenger. This can promote inclusive design practices by tailoring the display operation to the user capabilities by adequately configuring the "software-based" intent prediction algorithms and pointing facilitation schemes.

In this paper, we address the problem of identifying the most suitable scheme for facilitating the selection of the predicted interface component whilst driving. This human factor aspect is crucial for the deployment of the predictive touch technology in automotive. The selection facilitation functionality belongs to the Facilitation Scheme module in Figure 1. It involves altering the interface, for instance highlighting the predicted item, and then triggering the selection action. The users receive visual feedback on their input as the interface typically changes and the Graphical User Interface (GUI) page updates with each selection action. Other feedback modalities can be explored, e.g. audible (Ahmad et al., 2016b) or mid-air haptic (Shakeri, Williamson \& Brewster, 2018), however this is outside the scope of this article.

Here, the studies are conducted to establish the most effective selection facilitation scheme(s) for predictive touch as described in Figure 2. First, a set of eight viable schemes in an automotive environment are unveiled in a brainstorming session which is followed by an expert workshop study to identify the three highest rated ones. They were:

1. Immediate mid-air selection: The system autonomously auto-selects the highlighted predicted item on behalf of the driver without delay;

2. Hover/dwell: The driver's pointing finger hovers over the intended, highlighted, interface item for a finite duration of time;

3. Steering wheel button press: Driver presses an easily accessible button on the steering wheel to select the highlighted predicted GUI component.

These three selection facilitation schemes were subsequently evaluated in a study carried out in a mid-fidelity simulator with 24 participants using a prototype predictive touch system that operates seamlessly in real-time. The range of collected objective and subjective measures showed that immediate mid-air selection is the most promising facilitation scheme for a predictive touch system used by a driver, i.e. in a split attention scenario. 
Figure 1. System block diagram showing an in-car touchscreen, partial 3D pointing-finger trajectory (black solid line) available at the current time instant $t_{k}$ with tracked locations (crosses), future pointing trajectory (red dotted line) and intended on-screen destination (red circle)

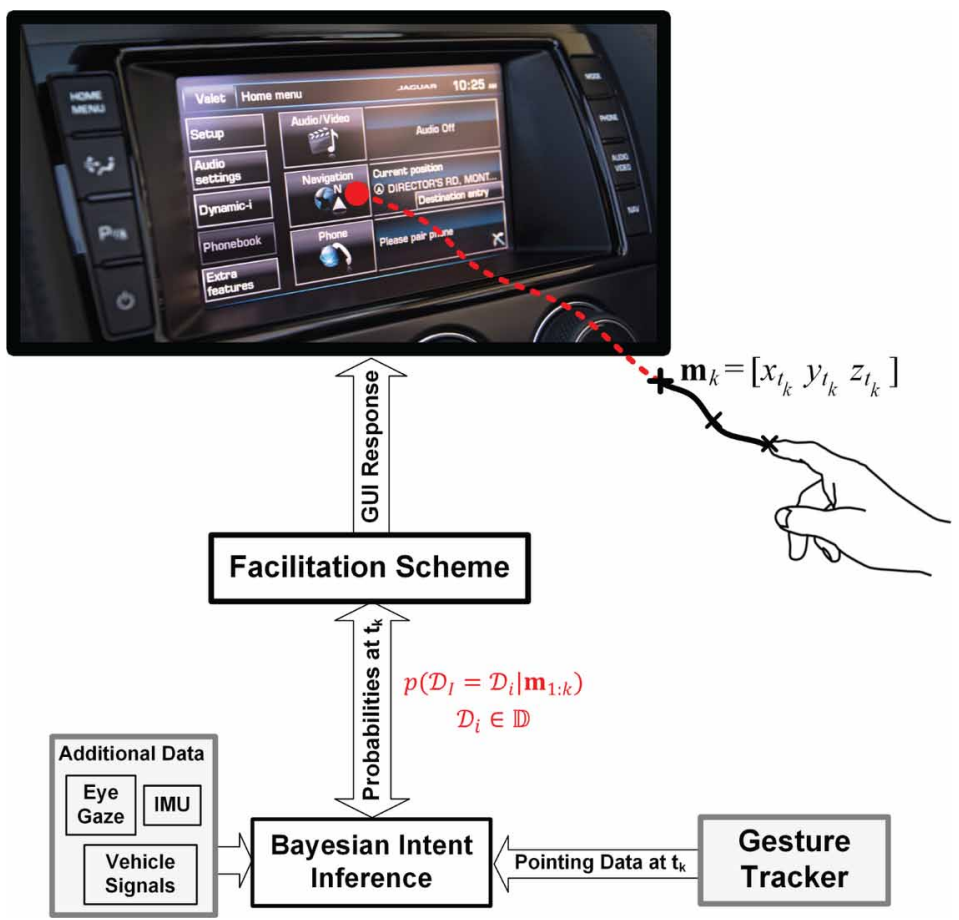

Figure 2. Studies in this paper to evaluate pointing facilitation schemes for predictive touch

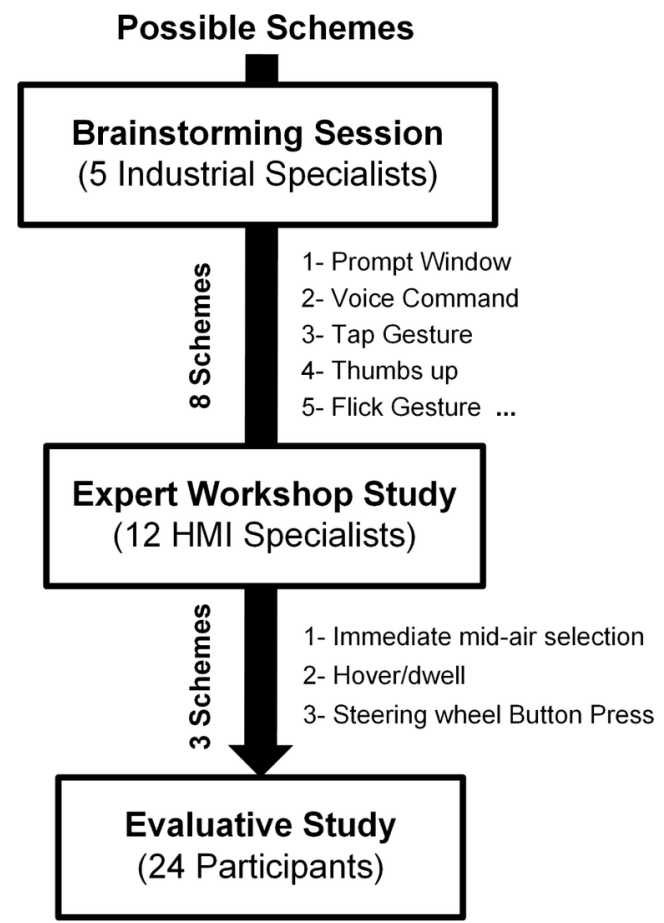




\section{BACKGROUND AND RELATED WORK}

According to the human motor movement model, Fitts' law, the index of difficulty $\left(\mathcal{I}_{D}\right)$ and the movement time $\left(T_{P}\right)$ for acquiring an interface component are given by:

$\mathcal{I}_{D}=\log _{2}(1+L / W), T_{P}=a+b \mathcal{I}_{D}$

such that W and L denote the width of the target GUI item and its distance from the starting position of the pointing apparatus, respectively; $a$ and $b$ are usually set empirically (Soukoreff \& MacKenzie, 2004). The selection task can therefore be simplified and expedited by increasing the target size (larger W) or moving it closer to the pointing apparatus (smaller L); these observations motivated basic pointing facilitation strategies in HCI (McGuffin \& Balakrishnan, 2005). Since a typical graphical user interface, for example an infotainment menu, contains several selectable items, a facilitation strategy should be preceded by a predictor to identify the intended on-screen icon, i.e. which icon(s) to expand, drag, etc. Hence, the problem of inferring the endpoint of a pointing movement has been addressed in several HCI studies, e.g. (Murata, 1998; McGuffin \& Balakrishnan, 2005; Pasqual \& Wobbrock, 2014), to name a few. However, the majority of these studies consider methods based on pointing on a computer monitor via a mouse or mechanical device to acquire a GUI icon in 2D using predictive models that are not pertinent for free hand pointing gesture in $3 \mathrm{D}$, e.g. interactions with in-vehicle displays, as shown by Ahmad et al. (2016a) and Ahmad et al. (2017).

Unlike previous studies on predictive touch which focused on devising intent prediction algorithms from pre-recorded data (Ahmad et al., 2016a), here the selection facilitation aspect of this HMI technology is considered. The evaluation study in this paper also uses a prototype system that runs in real-time with no visible delay in the system response. It examines various selection schemes and the system employs an automotive-grade propriety gesture tracker which does not constrain the driver's interaction style or area contrary to the tracker used in (Ahmad et al., 2016b). This paper expands on (Ahmad et al., 2018), e.g. incorporating visual behavior measures, and presents a more detailed analysis of collected data.

It should be noted that predictive touch is not a pointing or ray-casting (e.g., pointing at far away items) approach. It does not use the pointing finger/hand/arm location or orientation as a pointing apparatus, with or without a visual feedback on the pointing position (e.g., via projecting a cursor or crosshair on the GUI). For instance, it does not rely on the pointing finger(s) simply entering a predefined virtual interaction region in 3D space or casting a ray into the distant interface (e.g., as per the pointing finger-arm, possibly combined with head or eye-gaze, direction-orientation) to select a corresponding GUI component as with the mid-air pointing approaches in (Plaumann, Weing, Winkler, Müller \& Rukzio 2018; Mayer, Schwind, Schweigert \& Henze 2018; Roider \& Gross, 2018). Therefore, unlike such deterministic techniques, the studied predictive technology in this paper intrinsically depends on a probabilistic Bayesian framework that permits predicting the user intent from the tracked pointing movements in 3D and other data sources.

Additionally, predictive touch utilizes natural pointing gestures, that enable the user to acquire (point and select) various interface components without any learning overheads. This is contrary to classical gesture-recognition-based interactions, which have various well-established benefits and led to the proliferation of gesture trackers in automotive (Zhang \& Angell, 2014; May, Gable \& Walker, 2017; Shakeri, Williamson \& Brewster, 2018). However, they require the user to pre-learn particular gesture shapes to operate certain vehicle functions and the interaction area is often limited to a predefined small region where the recognition is robust. Inevitably, it can become more difficult or overwhelming for some users to remember the entire "symbolic" gestures set as the number of gesture-controlled functions increases. Nonetheless, the popularity of gesture recognition solutions 
amongst the major OEMs enabled predictive touch as a technology that can utilize an existing invehicle gesture tracker that can accurately and reliably track the pointing movement in 3D.

Finally, a selection strategy is typically an integrated part of most touch screen interface designs and numerous established criteria exist. They are based on multiple finger touch actions such as: location of on-screen mouse up or down events; hover time; double tap; pressure applied during a touch action and others (Zhai, Kristensson, Appert, Anderson \& Cao, 2012). However, predictive touch presents a new mode of interaction where the user does not need to touch the display to select an interface component. Therefore, many selection strategies for touch-based interaction are not suitable for the predictive HMI technology.

\section{EXPERT WORKSHOP STUDY}

\section{Identifying Potential Facilitation Schemes}

In order to limit the considered schemes to those realizable in an automotive setting under typical practical hardware and software constraints, five industrial specialists participated in a session and brainstormed several ideas. The participants were: a) three industrial human factors specialists, b) one software engineer and c) one interface designer. No demographic data was collected. At the end of this session, eight valid selection facilitation schemes for an in-vehicle predictive touch system were identified, none of which entail the user physically touching the display. As well as the three previously described schemes, i.e. immediate mid-air selection, press button on steering wheel (instrumentation is depicted in Figure 3) and hover/dwell, the other five schemes were:

- Prompt: A prompt screen is produced to inform the user that the highlighted interface item has been predicted as the intended on-screen item of the current pointing-selection task. The participant is then required to confirm their selection by pressing on "Confirm" or "Return" icons on the prompt window;

- Voice command: The predicted interface item is highlighted and the participant is required to confirm by a voice command, i.e. say "Select", to execute the selection action;

- Tap gesture: The participant makes a mid-air tap motion over the GUI item to select it, without touching the screen;

- Thumbs up gesture: Predicted GUI item is highlighted and the user makes a thumbs up hand gesture to execute the selection action;

- Flicking gesture: The participant makes a flicking side-ways motion of the hand to select the highlighted predicted GUI icon.

Figure 3. Instrumented button on the steering wheel for the button press selection scheme

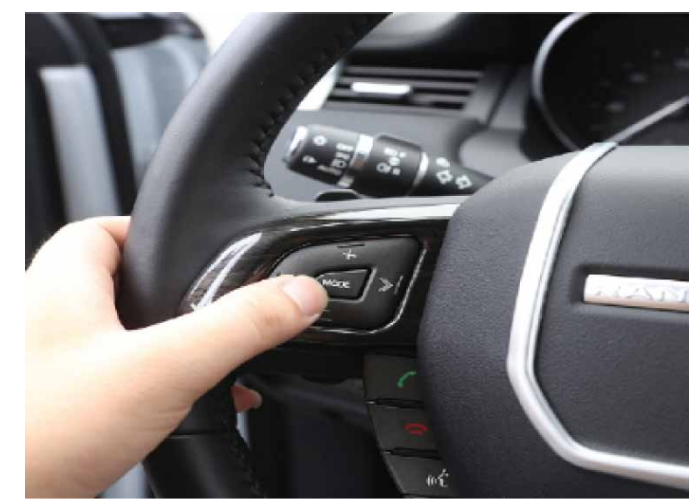




\section{Expert Workshop Study and Outcome}

Twelve Human Machine Interface (HMI) researchers, of which six were industrial human factor specialists, participated in the workshop. The objective of the workshop was to establish the three most promising pointing facilitation schemes for an in-vehicle predictive touch system, out of the eight identified valid schemes (see Figure 2).

At the start of the workshop, organizers described the predictive touch concept, its various modules and potential facilitation schemes in a neutral fashion. Each of the participants then:

1. Ranked the three they perceived as the most promising approaches (first choice being the most promising one), such that each rank was weighted as per: 1 ) first $=3$ points, 2 ) second $=2$ points and 3) third = 1 point;

2. Described the pros and cons of each facilitation scheme, with rationale and suggestions.

Table 1 gives the ranking scores for each of the eight considered selection facilitation schemes from all 12 participants. It also outlines the pros and cons of each scheme as stated by the participants. It can be seen in Table 1 that the three highest ranked approaches were: 1) immediate mid-air selection, 2 ) hover/dwell and 3) pressing a button on the steering wheel. These were selected for subsequent evaluation in the study. Some of the identified disadvantages of the other schemes further reinforced the rationale behind rejecting them for a practical in-vehicle system. For instance, a voice command can potentially conflict with listening to music or speaking to other vehicle occupants. Adding a prompt window can add several steps to a single pointing-selection task. Interestingly, some of the disadvantages of the three selected schemes in Table 1 were reflected in the results of the evaluation study described next.

\section{EVALUATION STUDY: METHOD}

\section{Participants: Demographics and Other Relevant Information}

Twenty-four participants (12 females) were recruited from the general public through an external agency. Whilst all had a valid UK driving license, participants were split into three age groups: 1) $18-38$ years $(29.2 \%), 2) 39-59$ years $(41.7 \%)$, and 2) 60+ years $(29.2 \%)$. Table 2 summarizes their demographics and related information.

Table 1. Pros and cons as well as ranking scores of the considered selection facilitation schemes

\begin{tabular}{|l|l|l|c|}
\hline \multicolumn{1}{|c|}{ Facilitation Scheme } & \multicolumn{1}{c|}{ Pros } & \multicolumn{1}{c|}{ Cons } & Ranking Score (Points) \\
\hline Immediate mid-air & $\begin{array}{l}\text { Fast and may not require } \\
\text { eyes off road time }\end{array}$ & $\begin{array}{l}\text { Possibility of frequent false } \\
\text { selections }\end{array}$ & 16 \\
\hline Hover/Dwell & - & $\begin{array}{l}\text { Increased workload with } \\
\text { dwell time can be too long }\end{array}$ & 13 \\
\hline Button Press & Less false selections & - & 16 \\
\hline Prompt & - & $\begin{array}{l}\text { Adds too many steps to } \\
\text { entry }\end{array}$ & 4 \\
\hline Voice Command & - & $\begin{array}{l}\text { Passenger presence and } \\
\text { music considerations }\end{array}$ & $11 / 1 / 5$ \\
\hline Tap/Thumb-up/Flick & - & $\begin{array}{l}\text { Conflicts with predictive } \\
\text { touch intent inference and } \\
\text { limits successive selections }\end{array}$ & \\
\hline
\end{tabular}


Table 2. The participants' demographic information

\begin{tabular}{|l|c|c|c|}
\hline & Mean & Minimum & Maximum \\
\hline Age (years) & 47 & 23 & 75 \\
\hline Driving experience (years) & 29 & 5 & 59 \\
\hline Miles per year (miles) & 14,375 & 3,000 & 30,000 \\
\hline
\end{tabular}

Additionally, participants reported that they drove the following diverse range of car models: Jaguar (2), BMW (5), Porsche (1), Audi (4), Land Rover (4), Mercedes (7) and Volvo (1). They were also queried before the study on their preferred: handedness of control and the most common start location of their pointing hand when interacting with the in-vehicle display on the center console. Their responses are given in terms of percentage of participants in Table 3. It is evident from the table that potential users of a predictive touch can start their freehand pointing movement from various locations. Therefore, it was deemed critical that the gesture tracker used had a wide coverage to support naturalistic interactions. Hence, a proprietary automotive-grade tracker was employed in this study.

\section{Conditions: Selection Facilitation Schemes}

Based on the predicted on-display destination of the tracked movement, the prototype predictive touch system implemented one of the following three selection facilitation schemes: 1) immediate mid-air auto-selection of the highlighted item without delay, 2) hover/dwell over the highlight predicted item for 2 seconds to trigger the selection action, and 3) a button press on the steering wheel. These were the tested conditions in this study. The 2 seconds dwell duration was set after pilot trials to minimize successive unintentional erroneous selections.

\section{Predictions}

- Immediate mid-air selection would expedite the interactions, would be the highest rated in terms of usability, and require the minimum level of visual attention;

- However, dwell would be the most demanding scheme in the split attention scenario;

- The selection facilitation scheme would not have a notable impact on the driving performance, due to the driver prioritising the driving task, and the relative simplicity of the LCT task.

\section{Apparatus}

A demonstration of the overall experimental set-up, apparatus, is shown in Figure 4. Here, the lefthand drive configuration was used without a HUD.

Table 3. Percentage of participants in relation to handedness of control and pointing start location

\begin{tabular}{|c|c|c|c|}
\hline \multicolumn{4}{|c|}{ Use left-hand drive (\%) } \\
\hline Never & Rarely & Sometimes & Often \\
\hline 16.7 & 41.7 & 29.2 & 12.5 \\
\hline \multicolumn{4}{|c|}{ Common start location of the pointing hand/arm (\%) } \\
\hline Arm rest & Steering wheel & Gear Stick & Lap \\
\hline 20.8 & 62.5 & 12.5 & 12.5 \\
\hline
\end{tabular}


Figure 4. Example simulator with gesture tracker mounted on the car roof (grey rectangle)

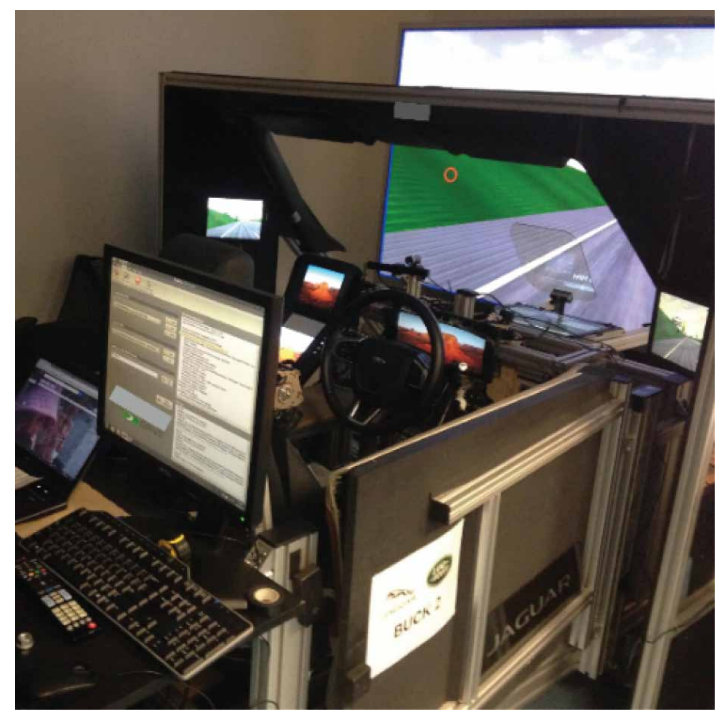

\section{Driving Simulator and Lane Change Test}

This evaluation study was conducted in a fixed mid-fidelity left hand drive simulator. The primary driving task was the Simulated Lane Change Test (LCT) as per the ISO 26022. Road signs, separated by unequal distances, appeared randomly instructing the driver to change between the three present lanes on a closed circuit with bends of varying radii; no other road users were present. Participants were required to maintain a nearly fixed speed of $60 \mathrm{~km} / \mathrm{h}$. A drive covered 10 tracks, which consisted of mainly straight sections of the circuit and hence resembling motorway-style driving. Each track included 18 lane changes and lasted for approximately 3 minutes.

\section{Display, Interface and Interaction Tasks}

A 13.3 inch touch screen with a resolution of $1920 \times 1080$ pixels was mounted to the simulator vehicle dashboard, central console, and displayed a newly designed JLR proprietary automotive interface. The exact GUI is commercially sensitive. However, a description of it is provided here, particularly in relation to the two interaction tasks used in this study.

The interface Home screen had three icons, including "Media" and "Contacts" icons, each sized $559 \mathrm{px} \times 330 \mathrm{px}$. Four fixed shortcut icons, $120 \mathrm{px} \times 120 \mathrm{px}$ each, were always present on the lefthand side of the interface to allow quick navigation to other menu options. Figure 5 shows the selection sequences of the two considered interaction tasks, which both started at the Home page. They were:

1. Change music track: Once the "Media" icon on the Home page was selected, the display presented the title and an image as well as five buttons including loop, rewind track, play/pause, forward track and random play track. The sizes of these selectable GUI icons were similar to those of the shortcuts. The displayed interface updated once a selection was made;

2. Dial a phone number: Once the "Contacts" button on the Home page was selected, the display presented a screen consisting of three main areas: 1) the shortcuts on the far left side, 2) a complete list of the available contacts displayed to the right of shortcuts, and 3) on the far right a number keypad which consisted of buttons for numbers 0-9 (240px × 120px), “*”, “\#”, "Delete" and "Dial" icons. On selecting a number, it was immediately shown in the keypad display. Once the phone number entry was complete, "Dial" icon was selected and the interface presented a prompt to show the dialing action. 


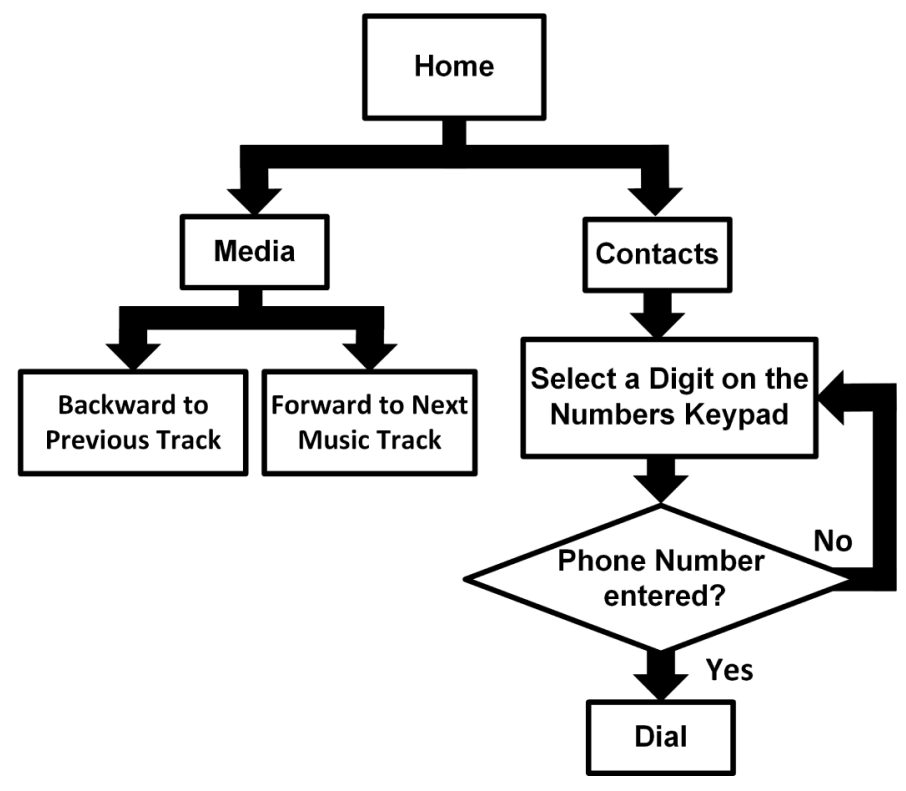

\section{Predictive Touch Prototype: Gesture Tracker and Predictor}

Since the main purpose of this study was to evaluate the most suitable selection facilitation scheme for predictive touch, a simple configuration of the system, in terms of data sources and prediction algorithm, was applied here. For instance, no eye-gaze and additional data were used. The employed prototype ran in real-time, implementing the predictive touch functionalities (for example, tracking, prediction and selection facilitation scheme) in real-time without any visible delay.

\section{Automotive-Grade Tracker}

A proprietary automotive-grade gesture tracker was mounted to the ceiling of the simulator car, facing downwards as depicted in Figure 4. This gave the tracker a wide coverage area incorporating the entire potential interaction area as in Table 3, for example steering wheel, lap, gear-stick and arm-rest to display regions. It continuously tracked the freehand pointing movements in $3 \mathrm{D}$ at a rate of up to $60 \mathrm{~Hz}$ and with an accuracy of a few millimeters. It provided the 3D Cartesian coordinates of the pointing finger-tip in 3D. For instance, $m_{k}=\left[\begin{array}{lll}\hat{x}_{t_{k}} & \hat{y}_{t_{k}} & \hat{z}_{t_{k}}\end{array}\right]^{\prime}$ denotes the sensory measurement at the time instant $t_{k}$ and thereby the partial pointing trajectory available to the prediction module is $m_{1: k}=\left\{m_{1}, m_{2}, \ldots, m_{k}\right\}$ pertaining to the sequential time instants $\left\{t_{1}, t_{2}, \ldots, t_{k}\right\}$. It was noted that data sorting and association are often needed prior to attaining the $m_{1: k}$ used by the predictor.

Intent Prediction and Decision Criterion

The intent inference module estimates the probability of each of the selectable interface icons being the intended on-screen destination based on the available sensory observations $m_{1: k}$ at $t_{k}$. Within the proposed Bayesian framework, these probabilities can be expressed by:

$$
p\left(\mathcal{D}_{I}=\mathcal{D}_{i} \mid m_{1: k}\right) \propto p\left(m_{1: k} \mid \mathcal{D}_{I}=\mathcal{D}_{i}\right) p\left(\mathcal{D}_{I}=\mathcal{D}_{i}\right), \mathcal{D}_{i} \in \mathbb{D}
$$


where $\mathbb{D}=\left\{\mathcal{D}_{i}: i=1,2, \ldots, N\right\}$ is the set of $N$ selectable GUI items and $\mathcal{D}_{I}$ is the true intended on-display endpoint. The term $p\left(\mathcal{D}_{i}=\mathcal{D}_{I}\right)$ is the prior on the $i^{\text {th }}$ selectable item and it can obtained from contextual or semantic information, frequency of use, interface design, additional sensory data such as eye-gaze, etc. A number of effective low complexity probabilistic models that are amenable to real-time implementations were proposed for predictive touch within a generic Bayesian inference framework, see (Ahmad et al., 2016a; Ahmad et. al., 2017). A few lead to a Kalman-filter-type implementation of the intent prediction routine, namely to estimate the likelihoods in (1), i.e. $p\left(m_{1: k} \mid \mathcal{D}_{I}=\mathcal{D}_{i}\right)$ for all interface components $\mathcal{D}_{i} \in \mathbb{D}$. Here and for simplicity, the following intuitive model is utilized:

$\operatorname{Pr}\left(\mathcal{D}_{I}=\mathcal{D}_{i} \mid m_{1: k}\right)=\mathcal{N}\left(m_{k} ; d_{i}, \sigma_{N N}^{2}\right)$

whilst $d_{i}=\left[\begin{array}{lll}d_{i, x} & d_{i, y} & d_{i, z}\end{array}\right]^{\prime}$ is the 3D location of the $i^{\text {th }}$ selectable interface item $\mathcal{D}_{i}, \mathcal{N}(e ; a, B)$ is a multivariate normal distribution with mean $a$ and covariance $B$. This approach thereby designated a high probability to the selectable interface icon that is close to the location of the pointing finger and vice versa, with the covariance $\sigma_{N N}^{2}$ in (2) dictating prediction certainty level. It also implicitly assumed that all icons had equal priors $p\left(\mathcal{D}_{I}=\mathcal{D}_{i}\right)=1 / N, \mathcal{D}_{i} \in \mathbb{D}$.

Based on the calculated probabilities and before applying a selection facilitation scheme, the system uses a decision criterion to determine the intended on-display endpoint at time $t_{k}$. In this study, the prototype system decided that a selectable interface item $\mathcal{D}_{i} \in \mathbb{D}$ was the intent of the pointing task if its calculated probability $p\left(\mathcal{D}_{I}=\mathcal{D}_{i} \mid m_{1: k}\right)$ exceeded a predefined threshold $\gamma$ for $L p$ successive predictions. This is in order to minimize false positives, i.e. erroneously unintended selections. The values of $\gamma$ and $L p$ were empirically chosen given the GUI design; they trade-off early predictions for false positives. Nevertheless, within the introduced Bayesian framework, this decision process can result from minimising $\mathbb{E}_{\mathcal{D}_{I}}\left[\mathcal{C}\left(\mathcal{D}^{*}, \mathcal{D}_{I}\right) \mid m_{1: k}\right]$ where $\mathcal{C}\left(\mathcal{D}^{*}, \mathcal{D}_{I}\right)$ is the cost function of deciding that $\mathcal{D}^{*}$ is the endpoint given that $\mathcal{D}_{I}$ is the true intended destination.

\section{Recording Visual Behavior}

A front facing GoPro webcam recorded the participants' actions while performing the driving and interaction tasks. Gaze and head pose data was extracted using the open-source tool OpenFace 2.1.0 (Baltrusaitis et al., 2018). Subsequently, the mean number of glances at the touchscreen and duration per glance was obtained from the analysis of the eye-gaze direction. A Support Vector Machine (SVM) classifier with a Gaussian kernel was trained on 3585 labelled images from the study. Input data for the classifier were $3 \mathrm{D}$ vectors for each eye and head pose rotation. The model correctly classified $99.8 \%$ of true eyes-on-touchscreen (10-fold cross validation).

\section{Performance Measures: Dependent Variables}

\section{Objective Measure}

- Lane deviation: Represented the driving performance and was measured according to the ISO 26022, for instance, deviations from an adaptive lane keeping model were estimated;

- Task Completion Time (TCT): Was the total duration (in seconds) of completing an interaction task, i.e. change music or dial a phone number, which encompassed several point-select actions;

- Glance count (GT) and Durations (GD): Was the number and duration (in seconds) of off-road glances during the interaction tasks, e.g. glancing at the display. 


\section{Subjective Measures}

- The NASA Task Load Index: The perceived workload index from a questionnaire completed by participants at the end of evaluating each condition (Hart \& Staveland, 1988);

- Customized preferences questionnaire: Due to the limited time available with participants, a questionnaire adapted from the two existing usability questionnaires, SUS and PSSUQ, was used. It addressed the selection facilitation schemes four key attributes: complexity, effectiveness, easiness and overall satisfaction. Participants accordingly responded to the following four statements using a scale 1 to 5 such that 1 is strongly agree and 5 is strongly disagree:

- I found the selection method unnecessarily complex (i.e., complexity);

- I could effectively complete the tasks and scenarios using this selection method (i.e., effectiveness);

- Easiness: Overall, I am satisfied with how easy it is to use this selection method (i.e., easiness);

- Overall Satisfaction: Overall, I am satisfied with this selection method (i.e. overall satisfaction);

- Post-trial ranking: Thematic analysis of the overall rankings to govern design recommendations (Glaser \& Stauss, 2017).

\section{Procedure}

\section{Pre-Trials}

On arrival, each participant completed the consent form, pre-simulator sickness form and demographic questionnaire. After entering the simulator and adjusting the seat and steering-wheel to their preferences, the predictive touch prototype system operation was checked and calibrated by the experimenter. Participants were then provided with an introduction and informed that there were no restrictions on the freehand pointing movement start position or style when interacting with the display.

Each participant then undertook "training" drives to familiarize themselves with the study set-up, at least once with and without interacting with the display. They were clearly instructed to follow the rules of the road, maintain the maximum speed limit of $60 \mathrm{~km} / \mathrm{h}$ and drive the simulator as they would in the real-world. Prioritizing the driving and lane change tasks during the study was emphasized.

\section{Trials}

During the trials, each participant was asked to perform the lane change driving task, maintain the speed of $60 \mathrm{~km} / \mathrm{h}$ and complete one of the following two interaction tasks when instructed (see Figure 5):

1. Enter and dial a particular phone number, out of the set $1118,8111,1181$ and 1811 ; or

2. Choose and play a particular music track by scrolling through (forward/backward/rewinding) the available list of tracks.

An equal number of phone number entries and music track changes were executed. In an attempt to increase the difficulty level of the secondary task of interacting with the display, requests to complete a given interaction were issued as or just before a lane changing road sign appeared on the simulator screen.

This procedure was repeated once per tested condition, i.e. immediate mid-air selection, hover/ dwell and button press. Participants filled the NASA TLX and the preferences questionnaires after using each of the three selection facilitation schemes. The trials duration ranged from 60 to 90 minutes per participant. During the study, conditions, including the type of interaction task, were randomized and counterbalanced to control order effects for the repeated measures design. Throughout the trials participants were monitored for simulator sickness and breaks were introduced when simulator sickness was reported. 


\section{Post-Trials}

After completing all of the drives-interactions for the three evaluated selection facilitation schemes, each participant was asked to complete a post-trial ranking for qualitative analysis as well as fill in a simulator sickness questionnaire and post-consent form.

\section{RESULTS}

Figure 6 outlines the procedure followed here to determine the statistical significance and accept or reject the null hypothesis (i.e. no difference in the outcomes of the three facilitation schemes) for all collected performance measures (dependent variables), except for the eye-gaze data and qualitative analysis. For instance, if the one-way Analysis of Variance (ANOVA) indicated statistical significance amongst the three tested conditions for a given performance measure, the Tukey's HSD range test (95\% family-wise confidence interval) was carried out to confirm. Data from both interaction tasks (dial a phone number and change song) were aggregated for the analysis of all measures, except the TCT. The task completion times were analyzed separately to reveal statistical effects.

\section{Lane Deviation}

The means and standard deviations of the recorded lane deviations were: immediate mid-air selection (mean 1.12 and std 0.35), hover/dwell (mean 1.15, std 0.42) and button press (mean 1.04 and std 0.36). The boxplot in Figure 7 shows two outliners, which were eliminated from the ANOVA test. Whilst pressing a button on the steering wheel resulted in marginally better average-median driving performance, ANOVA results indicated no statistical significant between the three conditions and the null hypothesis was not rejected, $\mathrm{F}(2,63)=0.55, \mathrm{p}=0.58$.

\section{Task Completion Time}

The mean TCTs of changing the music track and entering a phone number tasks are shown in Figure 8 (a) for each of the three tested conditions. The error bar is one standard deviation. It can be seen that the immediate mid-air selection scheme had the lowest average TCT, particularly for the more difficult

Figure 6. Procedure for determining statistical significance

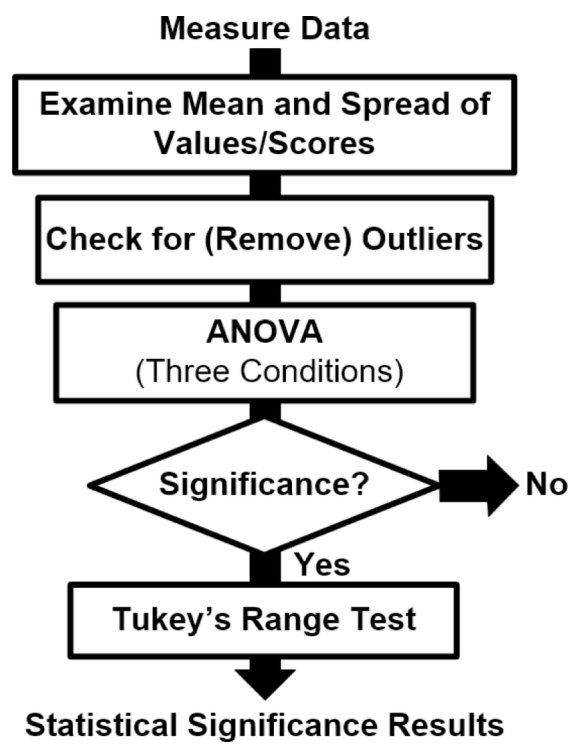


International Journal of Mobile Human Computer Interaction Volume 11 •Issue 3 • July-September 2019

Figure 7. Boxplot for the lane deviations measure

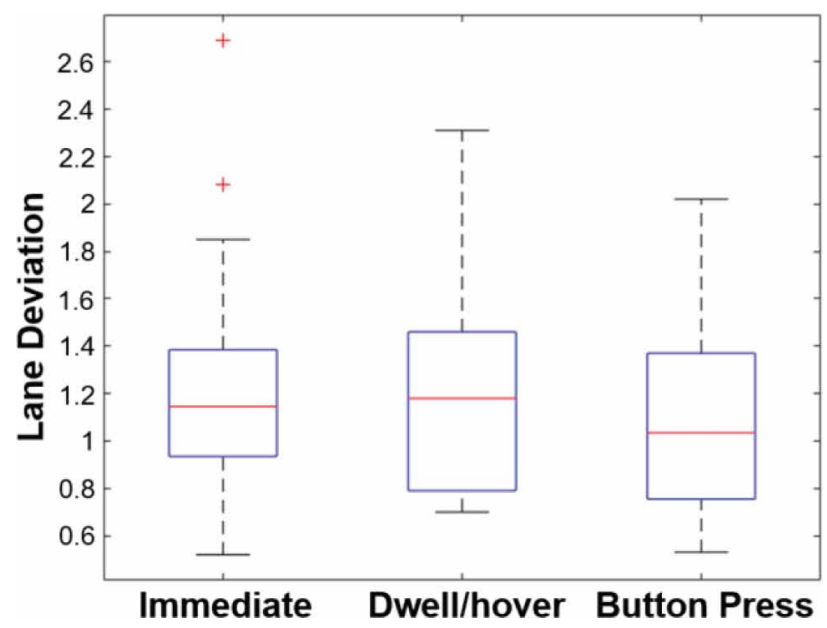

Figure 8. Analysis of the interaction task completion times. (a) Mean TCT and error bars are one standard deviation. (b)Tukey post-hoc analysis for dialing a phone number.

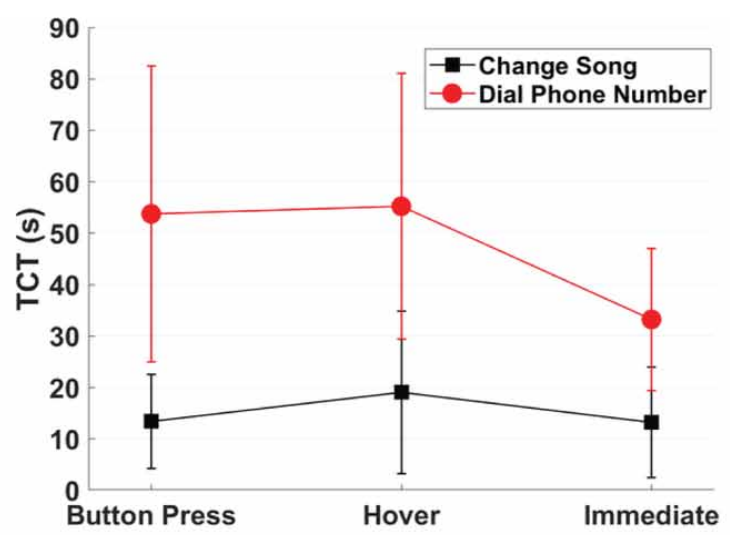

(a)

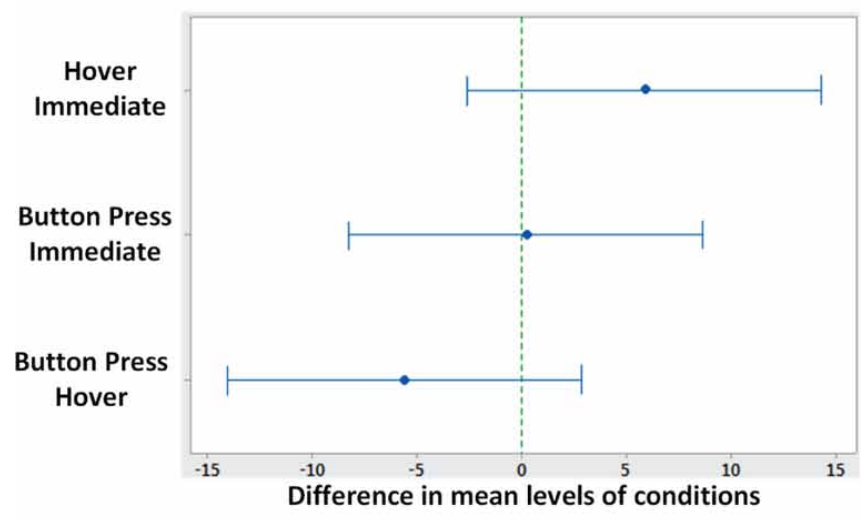

(b) 
interaction task of dialing phone number. It is over 38\% lower than the TCTs of the hover/dwell and button press approaches. Compared with changing the music track, dialing a phone number entailed, on average, notably more pointing-selection actions (i.e., perceptual-action cycles) and the numbers pad icon sizes were relatively small (Anderson, Matessa \& Lebiere, 1997; Soukoreff \& MacKenzie, 2004). Thus, dialing a phone number was more demanding and required significantly longer times as demonstrated in Figure 8. Whilst no outliers were detected in the collected TCT data, the ANOVA revealed:

- Changing music track: No statistical significant difference, $F(2,69)=1.77$ and $p=0.18$, and the null hypothesis was accepted;

- Dialing a phone number: There is statistical significance between the outcomes of the three conditions, $F(2,69)=6.46$ and $\mathrm{p}<0.01$, and the Tukey post hoc analysis results in Figure $8(\mathrm{~b})$ confirmed that the immediate mid-air selection scheme has a significantly different (lower) mean TCT. The null hypothesis was therefore rejected.

\section{Glance Count and Duration}

The number and duration of off-road glances are depicted in Figure 9; data from two of the participants had to be discarded due to poor quality camera output. Whilst the immediate mid-air selection had

Figure 9. Visual behavior analysis during the interaction tasks: (a) Number of off-road glances; (b) Duration of the glances

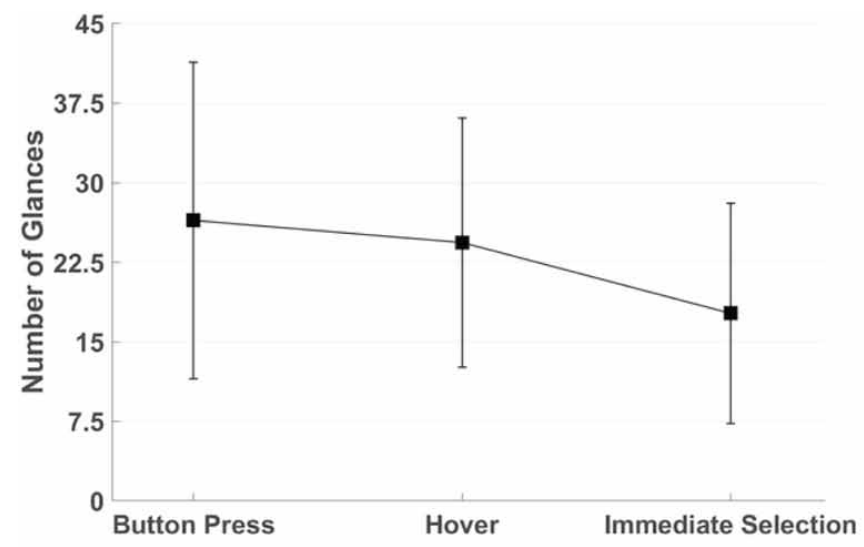

(a)

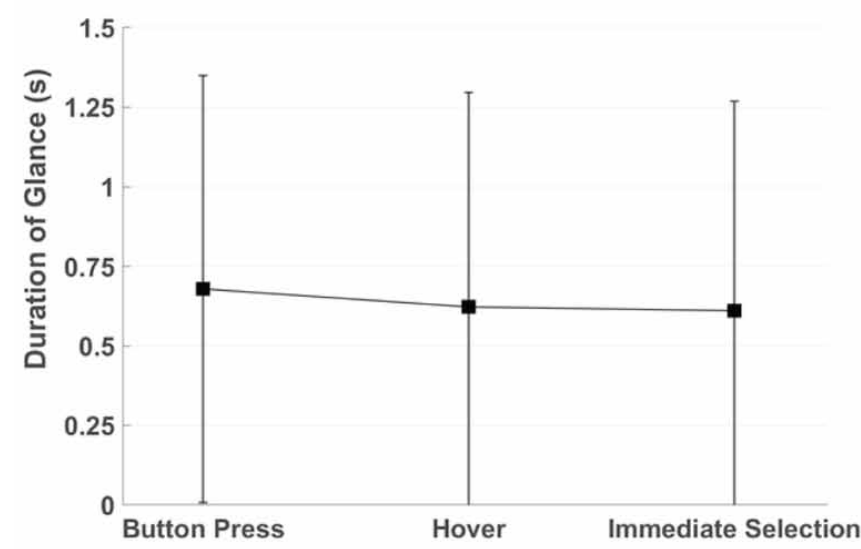

(b) 
a noticeably lower mean number of eyes-off-road glances followed by hover then button press. Although all conditions had similar duration of glances, immediate mid-air selection had a marginally lower average glances duration. The Friedman test for the number of glances measure showed a significant statistical difference between the conditions, $\mathcal{X}^{2}=60.59, \mathrm{p}<<0.05$. Post hoc Wilcoxon signed-rank tests confirmed that all pairwise comparisons were significant $(\mathrm{p}<0.05)$, except for hover/dwell and button press where $\mathrm{p}=0.56$. The null hypothesis was rejected for the glances count. This analysis confirms that immediate mid-air selection required substantially lower number of eyesoff-road glances, compared with hover (27.5\% less) and button press (33.2\% less). On the other hand, Friedman test revealed no statistical significant between the glances durations for the three facilitation schemes, $\mathrm{p}=0.63$, and the null hypothesis was accepted.

\section{NASA TLX}

Figure 10 displays the mean and standard deviation of the weighted NASA TLX scores for each of the three tested conditions. It can be noted from the figure that immediate mid-air selection had the lowest mean weighted subjective workload score; it is $18 \%$ and $20 \%$ lower than the hover/dwell and button press, respectively. Nonetheless and after checking that no outliers were present in the data, the ANOVA indicated lack of statistical significance between the outcomes of the three evaluated selection facilitation schemes, $F(2,69)=2.42$ and $p=0.09$. Consequently, the null hypothesis was accepted for this measure.

\section{Preferences Questionnaire}

The ratings for the four questions covering complexity, effectiveness, easiness and overall satisfaction were 1 to 5 such that 1 is strongly agree and 5 is strongly disagree. The mean and standard deviation (error bars) of the scores for all three selection facilitation schemes are displayed in Figure 11. Only the overall satisfaction question data had one outliner, whose data was eliminated from the subsequent statistical tests. Tukey post-hoc analysis in Figure 12 was conducted for the last three questions.

The mean scores of complexity for all conditions were similar and the null hypothesis was accepted; ANOVA $F(2,69)=0.195, \mathrm{p}=0.823$. The participants did not consider any of the evaluated facilitation schemes particularly complex to use and thus the scores did not noticeably differ between methods. The hover/dwell approach received the highest mean score (i.e., lowest rating) for effectiveness, whilst immediate mid-air selection had the highest rating (i.e., lowest score) as in Figure 11(b). The data spread for the effectiveness question is comparable for the three conditions. The one-way ANOVA showed significant statistical difference between

Figure 10. Mean and one standard deviation (error bars) of the weighted NASA TLX scores for three evaluated selection facilitation schemes

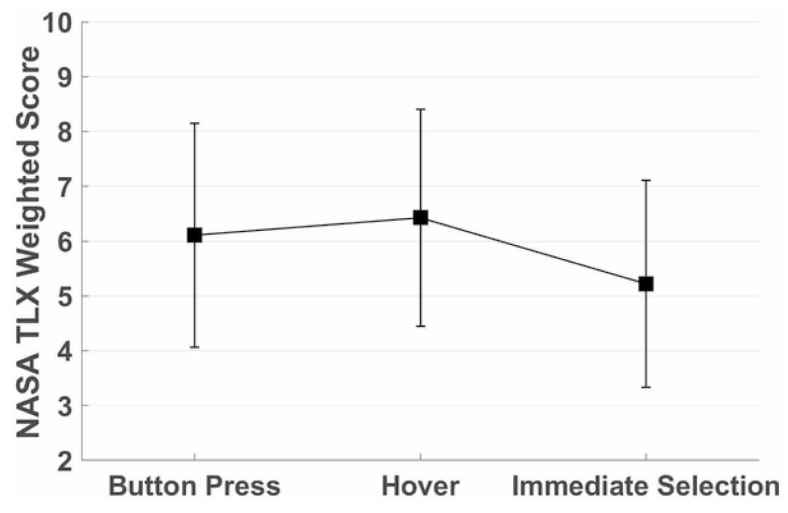


Figure 11. Mean and one standard deviation (error bars) of the ranking scores of the preferences questions: (a) Complexity; (b) Effectiveness; (c) Easiness; (d) Overall satisfaction

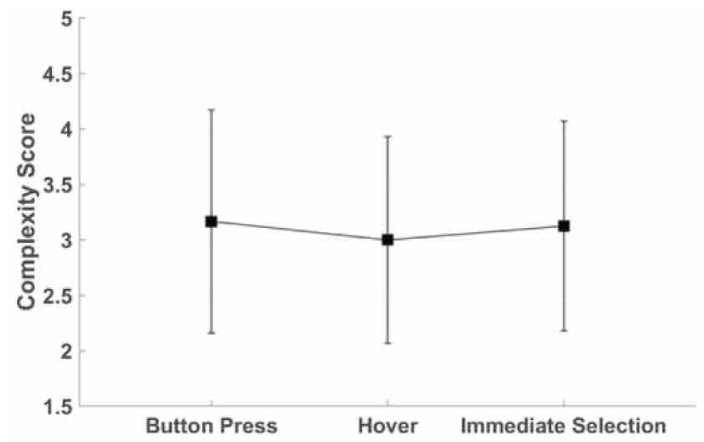

(a)

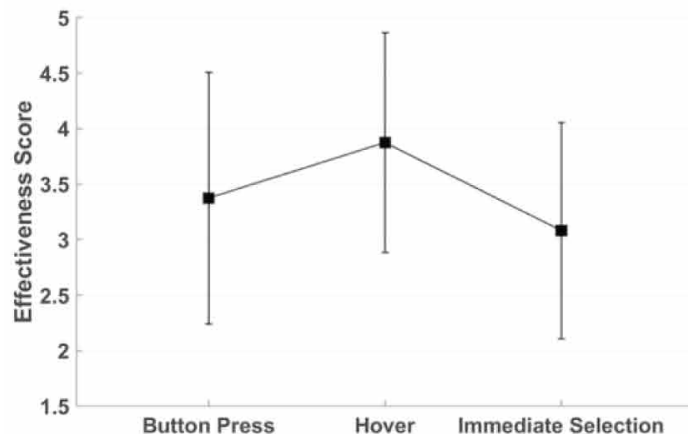

(b)

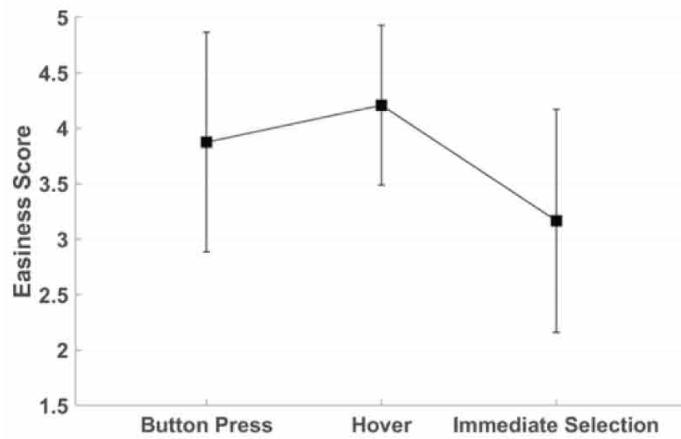

(c)

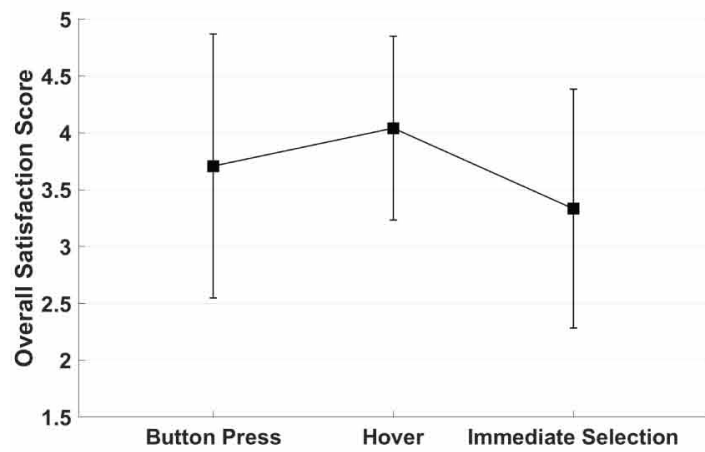

(d) 
conditions $F(2,69)=3.58, p<0.05$. A Tukey post-hoc analysis in Figure 12(a) clearly indicated that participants recognized that immediate mid-air selection could be used more effectively to complete the interaction tasks compared with hover/dwell, but less so than when compared to pressing a button on the steering wheel. It is also evident from Figure 11(c) that participants were favorable towards how easy it was to utilize immediate mid-air selection compared with the other two approaches. One-way ANOVA, $\mathrm{F}(2,69)=8.09, \mathrm{p}<0.01$, led to the rejection of the null hypothesis for the easiness question. Tukey post-hoc analysis in Figure 12(b) confirmed that participants agreed that the immediate mid-air selection was substantially easier to use compared with the hover/dwell and button press techniques. Participants felt that they were overall more satisfied with the immediate mid-air selection in comparison to the button press and hover/dwell conditions; the mean being visibly the smallest in Figure 11d. A one-way ANOVA revealed that there was a highly significant difference between conditions, $F(2,68)=3.749, \mathrm{p}<0.05$. The Tukey post-hoc analysis showed that immediate selection was markedly more satisfactory than hover, see Figure 12(c).

\section{Thematic Analysis: Post-Trial Ranking}

The rank frequency counts from the conducted post-trial qualitative interview analysis for each of the selection facilitation schemes are depicted in Table 4. Ranks 1 to 4 were used for clarity of exposition and the table only displays Rank 1 (best) and Rank 4 (least preferred).

The immediate mid-air selection scheme has substantially more overall total Rank 1 scores and lower Rank 4 scores. Only uncertainty is identified as Rank 4 theme for immediate mid-air selection, which was predictable since the participant had less control over when to trigger the selection action compared with pressing a button on the steering wheel and hover/dwell. The hover/dwell method had noticeably more Rank 4 scores. In general, the ranking in Table 4 confirms the pros and cons of each approach identified during the earlier expert workshop study.

In terms of the speed theme, the button press appears to have a higher score compared with immediate selection, despite the button press complexity theme in Rank 4. This is contrary to the previously analyzed objective measure of interaction task time for dialing a phone number. It is also expected that undertaking several secondary motor actions of pressing a button to execute the selections actions for a given interaction task should consume longer time. However, this could be explained by the fact that there was no clear non-visual feedback to confirm the execution of a selection action (e.g., an audible cue) for the immediate mid-air selection. This could have altered the participant's perception of the task duration, whereas they knew for sure that they did perform a selection action upon pressing the button on the steering wheel. It is also likely that a button press is a wellpracticed skill requiring little effort.

Figure 12. Tukey HSD post-hoc analysis for the preferences questions with ANOVA indicating statistical significance: (a) Effectiveness; (b) Easiness; (c) Overall satisfaction

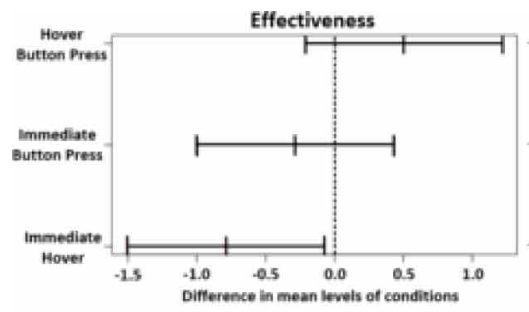

(a)

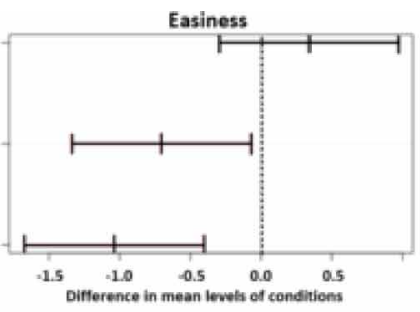

(b)

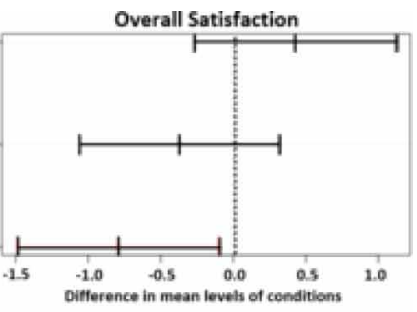

(c) 
Table 4. Participants rank frequency score only for ranks 1 and 4 from the analysis for all three evaluated conditions

\begin{tabular}{|c|c|c|c|}
\hline \multicolumn{2}{|c|}{ Rank 1} & \multicolumn{2}{|c|}{ Rank 4} \\
\hline Theme & Score & Theme & Score \\
\hline \multicolumn{4}{|c|}{ Immediate mid-air selection } \\
\hline Speed & 2 & Uncertainty & 1 \\
\hline Ease & 8 & & \\
\hline Effectiveness & 1 & & \\
\hline Distraction & 2 & & \\
\hline Eyes Off Road Time & 2 & & \\
\hline Familiarity & 1 & & \\
\hline \multicolumn{4}{|c|}{ Hover/Dwell } \\
\hline \multirow[t]{7}{*}{ Certainty } & 1 & Difficulty & 2 \\
\hline & & Selection & 3 \\
\hline & & Visibility & 1 \\
\hline & & Effectiveness & 1 \\
\hline & & Speed & 3 \\
\hline & & Accuracy & 2 \\
\hline & & Required Concentration & 1 \\
\hline \multicolumn{4}{|c|}{ Button Press } \\
\hline Speed & 4 & Tasks & 2 \\
\hline Ease & 3 & Speed & 1 \\
\hline Confirmation & 2 & Two Hand Requirement & 1 \\
\hline Control & 1 & Difficulty & 1 \\
\hline \multirow[t]{2}{*}{ Distraction } & 1 & Distraction & 1 \\
\hline & & Complexity & 2 \\
\hline
\end{tabular}

\section{DISCUSSION}

The summary of the key observations across all the collected measures are listed in Table 5. Overall, it is clear that the hover/dwell has limited benefits and is the least suitable for predictive touch according to several quantitative and qualitative measures. These results also confirm our predictions on the benefits of immediate mid-air selection in terms of the task completion time, visual demand and usability rankings.

The lack of significant statistical difference in the driving performance for the immediate selection and button press conditions can be attributed to the participants prioritizing the driving task as instructed by the experimenter. Nevertheless, the time required to accomplish the task of dialing a phone number clearly demonstrates that immediate mid-air selection is a notably more efficient approach compared to button press. We recall that dialing a phone number is noticeably more demanding when compared with the changing music track task.

Immediate mid-air selection has the lowest visual demand amongst the three assessed schemes, whereas no significant difference between the conditions is observed in terms of the duration of these 
Table 5. Summary of overall results

\begin{tabular}{|l|l|}
\hline \multicolumn{1}{|c|}{ Measure } & \multicolumn{1}{c|}{ Selected Key Results } \\
\hline Lane Deviation & No significance between conditions. \\
\hline Task Time & $\begin{array}{l}\text { Immediate mid-air selection task time is substantially lower compared with hover/dwell } \\
\text { and button press for entering a phone number. }\end{array}$ \\
\hline Visual demand & $\begin{array}{l}\text { Immediate mid-air selection requires the lowest number of off-road glances; no } \\
\text { significant difference between conditions for glances durations. }\end{array}$ \\
\hline Workload (NASA TLX) & No significance between conditions. \\
\hline Complexity Scores & No significance between conditions. \\
\hline Effectiveness Scores & $\begin{array}{l}\text { Immediate mid-air selection is more effective to complete the interaction tasks than } \\
\text { hover/dwell. }\end{array}$ \\
\hline Easiness Scores & $\begin{array}{l}\text { Ease scores show that participants were markedly more satisfied with immediate } \\
\text { selection in comparison to hover/dwell and button press. }\end{array}$ \\
\hline Satisfaction Scores & More satisfied with immediate selection (considerably more compared with hover/dwell). \\
\hline Rank Analysis & $\begin{array}{l}\text { Immediate mid-air selection was ranked significantly the highest for Rank 1 and lowest } \\
\text { for Rank 4 across several recognized themes. }\end{array}$ \\
\hline
\end{tabular}

glances. This can be predicted since priority was given to the primary task of driving as instructed, thereby minimising the duration of eyes-off-road glances. However, the frequency of these glances was driven by the implemented selection facilitation scheme where immediate selection did not demand multiple actions that necessitate visual attention. For instance, it did not involve monitoring the pointing finger position in 3D compared with hover/dwell which has the highest number of glances. Unexpectedly, button press on the steering had a marginally higher mean off-road glances count compared with hover/dwell. This can be explained by the need of several participants to look at the steering wheel prior to performing the button press action as confirmed by the recorded videos. This led to more off-road glances and challenged the assumption that button press is a well-practiced skill by all participants.

In terms of subjective measures from the preferences questions, immediate mid-air selection was rated substantially higher than the button press. The ranking analysis also confirmed this. For NASA TLX, the joint analysis of both interaction tasks together (changing song and dialing a phone number) might have led to the lack of significant statistical difference between the conditions. For example, the lower recorded mean weighted score and the notably low p-value of the ANOVA may have been due to aggregating the scores from both interaction tasks, which influenced the outcome. Since each participant filled the NASA TLX form after performing all interaction tasks under one of the three conditions, it was not possible to examine this further by studying data for each interaction task separately as with the TCT measure.

From a practical implementation perspective, the button press approach has two drawbacks compared to the immediate mid-air selection: 1) it requires a steering wheel instrumented with an easily accessible switch or button, dedicated to the predictive touch system, and 2) it cannot be used by passengers, e.g. to enter navigation information or configure media. On the other hand, immediate mid-air selection has no additional hardware requirements and its implementation does not necessitate introducing changes to legacy automotive HMI software architecture; it only entails sending a trigger selection signal to the existing interface software module. Overall, the data analyzed in this paper showed that immediate mid-air selection is, in general, the most promising selection facilitation scheme for the predictive touch technology. 


\section{CONCLUSION AND FINAL REMARKS}

Predictive touch is a novel HMI technology that enables the reliable interactions with in-vehicle displays, without the need to physically touch a surface to acquire an interface component. Hence, it constitutes touch-free, mid-air, control that uses (intuitive) freehand pointing gestures, without the need for learning symbolic gestures. Nevertheless, this HMI poses new challenges, such as selection facilitation strategies, which are well studied for more conventional approaches such as touch-based input on a screen or pad or using a mouse-cursor. The evaluation study in this paper illustrates that immediate mid-air selection is an effective pointing-selection facilitation scheme that best exploits the potential of the predictive touch technology. Conversely, a hover/dwell approach is the least suitable strategy as revealed by the objective and subjective measures. Interestingly, the immediate mid-air selection is particularly relevant to interacting with proliferating new display technologies in automotive, such as 3D projections and head-up displays, which do not have a physical surface. It also requires minimal modification to existing legacy automotive GUI software architecture with minimal display overheads. It is important to note that predictive touch is capable of (and was originally designed for) compensating for vibrations and accelerations in the moving vehicle due to road and driving conditions, within the proposed Bayesian filtering framework. In this respect it is unique and not comparable with conventional pointing (Plaumann, Weing, Winkler, Müller \& Rukzio 2018; Mayer, Schwind, Schweigert \& Henze 2018; Roider \& Gross, 2018) and gesture recognition approaches (Zhang \& Angell, 2014; May, Gable \& Walker, 2017).

This paper can motivate further future work to evaluate the overall predictive touch technology in more ecologically valid, naturalistic, driving settings and address the methodological limitations of the studies presented here. This includes on-road evaluations that incorporate the influence of road-driving conditions (e.g., vibrations and accelerations) on the pointing gestures, and undertaking more demanding driving scenarios compared with the LCT. The impact of providing the user with feedback on the completion of the interaction task, i.e. beyond visual as in this paper, need to be investigated. Additionally, more advanced intent predictors can be utilized, e.g. those that employ stochastic motion and measurements models to achieve early accurate inference results (Ahmad et al., 2017) and fuse more sensory data such as eye-gaze. The ranking analysis presented above only focused on the top and bottom ranks for ease of explication. Only a small number of performance measures and a customized questionnaire scores were also collected due to operational constraints. This invites more detailed qualitative analysis, exploring usability in greater depth with validated methods and capturing additional measures such as (objective) workload and driving performance metrics. 


\section{REFERENCES}

Ahmad, B. I., Hare, C., Singh, H., Shabani, A., Lindsay, B., Skrypchuk, L., \& Godsill, S. et al. (2018, September). Selection Facilitation Schemes for Predictive Touch with Mid-air Pointing Gestures in Automotive Displays. In Proceedings of the 10th International Conference on Automotive User Interfaces and Interactive Vehicular Applications (pp. 21-32). ACM. doi:10.1145/3239060.3239067

Ahmad, B. I., Langdon, P. M., Godsill, S. J., Donkor, R., Wilde, R., \& Skrypchuk, L. (2016b, October). You do not have to touch to select: A study on predictive in-car touchscreen with mid-air selection. In Proceedings of the 8th International Conference on Automotive User Interfaces and Interactive Vehicular Applications (pp. 113-120). ACM. doi:10.1145/3003715.3005461

Ahmad, B. I., Langdon, P. M., Godsill, S. J., Hardy, R., Skrypchuk, L., \& Donkor, R. (2015, September). Touchscreen usability and input performance in vehicles under different road conditions: an evaluative study. In Proceedings of the 7th International Conference on Automotive User Interfaces and Interactive Vehicular Applications (pp. 47-54). ACM. doi:10.1145/2799250.2799284

Ahmad, B. I., Murphy, J. K., Godsill, S., Langdon, P. M., \& Hardy, R. (2017). Intelligent interactive displays in vehicles with intent prediction: A Bayesian framework. IEEE Signal Processing Magazine, 34(2), 82-94. doi:10.1109/MSP.2016.2638699

Ahmad, B. I., Murphy, J. K., Langdon, P. M., Godsill, S. J., Hardy, R., \& Skrypchuk, L. (2016a). Intent inference for hand pointing gesture-based interactions in vehicles. IEEE Transactions on Cybernetics, 46(4), 878-889. doi:10.1109/TCYB.2015.2417053 PMID:25935053

Anderson, J. R., Matessa, M., \& Lebiere, C. (1997). ACT-R: A theory of higher level cognition and its relation to visual attention. Human-Computer Interaction, 12(4), 439-462. doi:10.1207/s15327051hci1204_5

Baltrusaitis, T., Zadeh, A., Lim, Y. C., \& Morency, L. P. (2018, May). OpenFace 2.0: Facial Behavior Analysis Toolkit. In 2018 13th IEEE International Conference on Automatic Face \& Gesture Recognition (FG 2018) (pp. 59-66). IEEE.

Bark, K., Tran, C., Fujimura, K., \& Ng-Thow-Hing, V. (2014, September). Personal Navi: benefits of an augmented reality navigational aid using a See-Thru 3D Volumetric HUD. In Proceedings of the 6th International Conference on Automotive User Interfaces and Interactive Vehicular Applications (pp. 1-8). ACM. doi:10.1145/2667317.2667329

Broy, N., Guo, M., Schneegass, S., Pfleging, B., \& Alt, F. (2015, September). Introducing novel technologies in the car: conducting a real-world study to test 3D dashboards. In Proceedings of the 7th International Conference on Automotive User Interfaces and Interactive Vehicular Applications (pp. 179-186). ACM. doi: $10.1145 / 2799250.2799280$

Glaser, B. G., \& Strauss, A. L. (2017). Discovery of grounded theory: Strategies for qualitative research. Routledge. doi:10.4324/9780203793206

Goode, N., Lenné, M. G., \& Salmon, P. (2012). The impact of on-road motion on BMS touch screen device operation. Ergonomics, 55(9), 986-996. doi:10.1080/00140139.2012.685496 PMID:22676650

Hart, S. G., \& Staveland, L. E. (1988). Development of NASA-TLX (Task Load Index): Results of empirical and theoretical research. Advances in Psychology, 52, 139-183. doi:10.1016/S0166-4115(08)62386-9

Harvey, C., \& Stanton, N. A. (2016). Usability evaluation for in-vehicle systems. CRC Press. doi:10.1201/b14644

Jæger, M. G., Skov, M. B., \& Thomassen, N. G. (2008, April). You can touch, but you can't look: interacting with in-vehicle systems. In Proceedings of the SIGCHI Conference on Human Factors in Computing Systems (pp. 1139-1148). ACM.

May, K. R., Gable, T. M., \& Walker, B. N. (2017, September). Designing an In-Vehicle Air Gesture Set Using Elicitation Methods. In Proceedings of the 9th International Conference on Automotive User Interfaces and Interactive Vehicular Applications (pp. 74-83). ACM. doi:10.1145/3122986.3123015

Mayer, S., Schwind, V., Schweigert, R., \& Henze, N. (2018, April). The Effect of Offset Correction and Cursor on Mid-Air Pointing in Real and Virtual Environments. In Proceedings of the 2018 CHI Conference on Human Factors in Computing Systems (p. 653). ACM. doi:10.1145/3173574.3174227 
McGuffin, M. J., \& Balakrishnan, R. (2005). Fitts' law and expanding targets: Experimental studies and designs for user interfaces. ACM Transactions on Computer-Human Interaction, 12(4), 388-422. doi:10.1145/1121112.1121115

Murata, A. (1998). Improvement of pointing time by predicting targets in pointing with a PC mouse. International Journal of Human-Computer Interaction, 10(1), 23-32. doi:10.1207/s15327590ijhc1001_2

Ohn-Bar, E., \& Trivedi, M. M. (2014). Hand gesture recognition in real time for automotive interfaces: A multimodal vision-based approach and evaluations. IEEE Transactions on Intelligent Transportation Systems, 15(6), 2368-2377. doi:10.1109/TITS.2014.2337331

Pasqual, P. T., \& Wobbrock, J. O. (2014, April). Mouse pointing endpoint prediction using kinematic template matching. In Proceedings of the SIGCHI Conference on Human Factors in Computing Systems (pp. 743-752). ACM. doi:10.1145/2556288.2557406

Plaumann, K., Weing, M., Winkler, C., Müller, M., \& Rukzio, E. (2018). Towards accurate cursorless pointing: The effects of ocular dominance and handedness. Personal and Ubiquitous Computing, 22(4), 633-646. doi:10.1007/s00779-017-1100-7

Roider, F., \& Gross, T. (2018, September). I See Your Point: Integrating Gaze to Enhance Pointing Gesture Accuracy While Driving. In Proceedings of the 10th International Conference on Automotive User Interfaces and Interactive Vehicular Applications (pp. 351-358). ACM. doi:10.1145/3239060.3239084

Shakeri, G., Williamson, J. H., \& Brewster, S. (2018). May the Force Be with You: Ultrasound Haptic Feedback for Mid-Air Gesture Interaction in Cars. In Proceedings of the 8th International Conference on Automotive User Interfaces and Interactive Vehicular Applications (pp. 113-120). ACM doi:10.1145/3239060.3239081

Soukoreff, R. W., \& MacKenzie, I. S. (2004). Towards a standard for pointing device evaluation, perspectives on 27 years of Fitts' law research in HCI. International Journal of Human-Computer Studies, 61(6), 751-789. doi:10.1016/j.jjhcs.2004.09.001

Zhai, S., Kristensson, P. O., Appert, C., Anderson, T. H., \& Cao, X. (2012). Foundational issues in touch-surface stroke gesture design - an integrative review. Foundations and Trends in Human-Computer Interaction, 5(2), 97-205.

Zhang, Y., \& Angell, L. (2014, September). Pointing towards future automotive HMIs: The potential for gesture interaction. In Adjunct Proceedings of the 6th International Conference on Automotive User Interfaces and Interactive Vehicular Applications (pp. 1-6). ACM.

Bashar I. Ahmad received the B.Eng. (Hons.) in Electronic Engineering and Ph.D. degrees from the University of Westminster, U.K. in 2007 and 2011, respectively. He is currently a Senior Research Associate in the Signal Processing and Communications (SigProC) Laboratory, Engineering Department, Cambridge University, U.K. and a fellow of Wolfson College, Cambridge. Prior to joining Cambridge University, he was a postdoctoral researcher in the Signal Processing and Communications group at Imperial College London, U.K. His research interests include theory and applications of statistical signal processing and machine learning, such as in intelligent vehicles, sensing systems, automation, data analytics, defense, behavior modelling, wireless communications, human computer interaction and others.

Chrisminder Hare; Human Factors Specialist at Jaguar Land Rover Harpreet Singh - Creative engineer and researcher with a strong background in Infotainment, Electronics, $\mathrm{HCl}$ and $\mathrm{HMI}$. Technical project leader in automotive domain, that involves applying human factors principles along with system design engineering to deliver new technology concepts to enhance user experience and safety for both tradition and future autonomous driving.

Arber Shabani - Experienced Human Factors Specialist with a demonstrated history of working in the automotive industry. At Jaguar Land Rover I have been involved on designing various innovative technologies for in-cabin experience based on the occupants' preferences, emotional experiences and cognitive behaviour. Skilled in UX and HMI user testing, design research, requirement definition, digital prototyping and concept development. Passionate about the future of automotive design and user research with a PhD degree focused in Human Centred Design from Brunel University London. 